\title{
Knifeless ultrasound brain surgery promises to cut risks, recovery time
}

\author{
n Cite as: CMAJ 2018 February 5;190:E151-2. doi: 10.1503/cmaj.109-5555
}

Posted on cmajnews.com on Jan.18, 2018.

$\mathrm{T}$ he future of brain surgery may look like the beauty parlors of the past, with patients in domed helmets chatting with their surgical team as they undergo knifeless operations.

This vision is becoming reality at the Sunnybrook Research Institute in Toronto, where neurosurgeon and scientist Dr. Nir Lipsman is pioneering the use of ultrasound to heat and destroy misfiring neurons, avoiding the risks of open surgery. The knifeless surgery holds promise for treating otherwise unmanageable mood disorders and dementia, Lipsman said at a brain health meeting at Sunnybrook. "This is a powerful potential tool and so far patients have been doing well."

The dream of harnessing ultrasound energy for neurosurgery goes back 50 years, he explained. But until recently, "the problem has been that the bone, the skull around the brain, absorbs and refracts that energy," making it difficult to use with precision.

Two things happened to overcome that challenge, Lipsman said. "First, was the coupling of ultrasound energy with magnetic resonance imaging, so that you can detect tissue temperature in the brain as you're heating," allowing surgeons to see lesions form in real time. Then came a helmet lined with more than 1000 ultrasound transducer elements. "We can steer each of those transducers onto discrete regions of the brain," enabling surgeons to target areas a few millimetres in size.

This "Holy Grail" combination of realtime feedback and precision allows surgeons to heat tissue just below the point of forming a lesion to "knock out" neurons temporarily, Lipsman said. Patients are awake and can be tested during surgery, "so if you're getting any side effects, you can stop heating and move your target without causing permanent damage."

Lipsman led a proof-of-concept study and coauthored a randomized trial that showed focused ultrasound therapy is

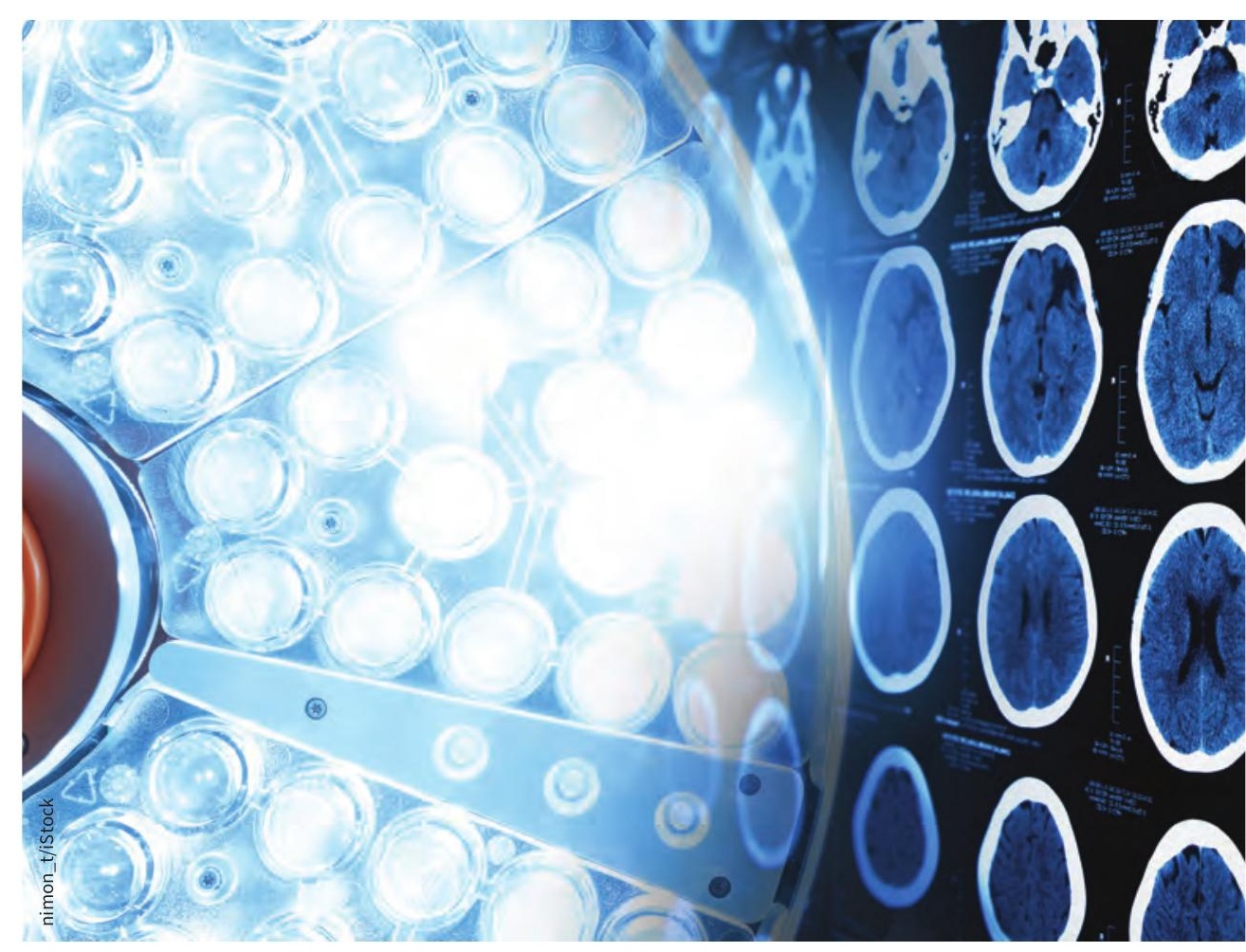

Researchers are investigating knifeless ultrasound brain surgery to treat mental health problems.

safe and effective in treating essential tremor, a common motion-control disorder. Within hours of treatment, in patients who previously couldn't feed or dress themselves, "the tremor is totally gone," he said. Adverse effects included sensory and gait disturbances. Sunnybrook has trials underway investigating the use of focused ultrasound in treating depression, obsessive-compulsive disorder and Alzheimer disease.

About a third of patients with mood disorders don't respond to drugs or therapy, making them potential candidates for surgical intervention, Lipsman said. Conven- 
In the case of Alzheimer disease, researchers will use the technology to open the blood-brain barrier temporarily to improve delivery of treatments to the brain. Sunnybrook researchers have already used the technique for brain tumours in humans to deliver chemotherapy that otherwise couldn't penetrate the blood-brain barrier. Now, they're developing a trial to open the blood-brain barrier in the right frontal lobe of patients with early-stage Alzheimer disease, said Lipsman. There's a potential the procedure may also open the brain to as yet unknown harm, "so if there's any chance a patient's sick or has an infection, we don't do it," he said. "If we can demonstrate that it's safe, we'll move on to other areas that are more eloquent and intimately involved in memory."

Lauren Vogel, CMAJ 